\title{
Strengthened Data Systems to Mitigate the Double Burden of Malnutrition: The Role of Stable Isotope Technique-Derived Nutrition Indicators
}

\author{
Victor O. Owino Theodora Mouratidou \\ Division of Human Health, Department of Nuclear Sciences and Applications, International Atomic Energy Agency, \\ Vienna, Austria
}

\section{Keywords}

Stable isotopes - Nutrition surveillance - Double burden of malnutrition $\cdot$ Global nutrition targets

\begin{abstract}
Indicators reflecting the double burden of malnutrition are rarely measured in nutrition surveys and are needed to strengthen national data systems. Indicators such as body composition reflect both metabolic response to undernutrition and obesity risk and nutrition-related noncommunicable diseases. Stable isotope techniques (SITs) provide accurate data on body composition, exclusive breastfeeding and vitamin A status that are otherwise problematic with routine methods. Integration of SIT-derived nutrition indicators in data systems could improve the design and evaluation of programmes focused on obesity prevention, food fortification and infant and young child feeding practices. The Working Group at the symposium considered "how SIT-derived nutrition indicators may be integrated into surveillance systems to strengthen data availability and capacity at national and regional levels". Practical considerations for the use of SITs include cost, sample size, rigorous training and logistics. It was concluded that SITs are best suited, at present, for use in subsamples of population surveys and for validating tools that can be scaled-up more easily in population surveys. In the long term, SITs could be applied to larger surveys following
\end{abstract}

potential innovations in more affordable, hand-held devices for analysis of stable isotope enrichment in the field and simpler specimen collection protocols.

(c) International Atomic Energy Agency 2019 Published by S. Karger AG, Basel

\section{Introduction}

There is a need for strengthening the collection of timely and accurate data to support the achievement of the Sustainable Development Goals (SDGs) 2 and 3 [1] and the commitments of the Rome Declaration on Nutrition. Despite considerable progress in measuring malnutrition (i.e., available databases for monitoring SDGs indicators 2.2.1 and 2.2.2 [2]), much remains to be done if the SDGs are to be met. The increasing prevalence of the double burden of malnutrition (DBM) poses an additional and complex measurement challenge, highlighting the need for robust and updated data systems and monitoring frameworks. For this reason, novel nutrition indicators are needed to better measure and understand expo-

The article is part of the Proceedings of the International Symposium on Understanding the Double Burden of Malnutrition for Effective Interventions organized by the International Atomic Energy Agency (IAEA) in cooperation with United Nations Children's Fund (UNICEF) and World Health Organization (WHO) (10-13 December 2018, Vienna, Austria).

\begin{tabular}{ll}
\hline KARGER & $\begin{array}{l}\text { ( International Atomic Energy Agency } 2019 \\
\text { Published by S. Karger AG, Basel }\end{array}$ \\
E-Mail karger@karger.com & $\begin{array}{l}\text { All rights reserved. } \\
\text { This is an Open Access article licensed under the terms of the Creative } \\
\text { www.karger.com/anm }\end{array}$ \\
$\begin{array}{l}\text { Commons Attribution 3.0 IGO License (CC BY 3.0 IGO)https://cre- } \\
\text { ativecommons.org/licenses/by/3.0/igo/\#). Usage, distribution and re- } \\
\text { production in any medium or format, even for commercial purposes, } \\
\text { is permitted, provided the original work is properly cited. }\end{array}$
\end{tabular}


sure-outcome interactions to best inform the design and improvement of activities responding to the DBM. The International Atomic Energy Agency (IAEA) sub-programme on nutrition encourages Member States to apply stable isotope techniques (SITs) to accurately measure nutritional indicators such as breast milk intake and exclusive breastfeeding, body composition, total energy expenditure, bioavailability and absorption of essential minerals (iron, zinc) and vitamin A body pool size as a measure of vitamin A status [3].

The adoption of SIT-derived nutrition indicators will lead to improvements in the assessment of critical indicators for better informed design and evaluation of national programmes on the prevention and control of programmes focused on obesity, food fortification and infant and young child feeding practices. The below-mentioned examples have used SITs and have informed policy by studying mechanisms and associations in a small number of participants. One study from Seychelles measured body fat using the deuterium oxide dilution technique and found that approximately half of 8- to 10-year-old schoolchildren had excess body fat [4]. However, the study results were compared to cut-offs for excess body fat based on anthropometry [5], highlighting the need for cut-offs based on stable isotopes. Stable isotope-derived data on body fat and energy expenditure were used in Chile to develop a preschool nutrition education and physical activity programme in response to a rapid rise in obesity among preschool children [6]. In Guatemala, the deuterium dose to the mother technique (DTM) was used to confirm self-reported breastfeeding practices among mothers [7]. As reported in other countries [8-10], exclusive breastfeeding was overestimated when assessed using maternal recall compared to that measured using DTM. Studies using SITs have also been used successfully to inform decision making involving food fortification with micronutrients, such as iron [11] and vitamin A [12].

\section{Can SITs Be Used in the Context of Large-Scale} Surveys?

Household survey programmes, such as the UNICEFsupported Multiple Indicator Cluster Surveys, National Micronutrient Surveys, the USAID-supported Demographic and Health Surveys (DHS) [13] and populationbased representative surveys, offer the possibility to collect data for better planning and design of mitigating interventions and support for monitoring and evaluation of interventions, programmes and policy changes to respond to the DBM. SIT-derived nutrition indicators could be integrated in such survey programmes to complement and strengthen national and regional responses to the DBM. SITs follow the same procedural steps involved in national surveys with due consideration for ethical practice [14, 15]. Examples of such nutrition indicators include adiposity (body fat mass) and lean body mass, exclusive breastfeeding and vitamin A status. Their use lends a great opportunity to collect information on nutrition indicators that are otherwise impossible to obtain with standard nutrition assessment methods. In particular, the ability to accurately measure excess body fat as an important risk of nutrition-related noncommunicable diseases [16-19] and the ability to measure breast milk intake and the exclusivity of breastfeeding $[3,20]$ are of profound public health relevance. Alternative indicators based on anthropometry (e.g., BMI) remain crucial for screening and categorising individuals based on risk of disease, but in contrast to body fat, they do not allow nuanced interpretation of underlying metabolic pathways [19, 21, 22].

Strategies for scaling up the use of stable isotopes in large population surveys have yet to be considered. Integrating the use of stable isotopes in large surveys was the focus of a Working Group (WG) at the International Symposium on the DBM "as part of an effort to strengthen data availability and capacity at national and regional levels to meet international commitments in the framework of the SDGs". The WG was attended by a diverse group of stakeholders from academia, government ministries, UN agencies and practitioners. Discussions were guided by the following points: (1) relevance of SITs in national surveillance programmes, (2) practical and financial considerations when choosing SIT-derived nutrition indicators, (3) processes to be followed for integration of SIT-derived nutrition indicators in national surveys and (4) potential challenges and opportunities, including linkages and partnerships. What follows is a brief summary of the input and outcomes of this WG.

\section{Proposed Strategy for Integrating SITs in Large Population Surveys}

To integrate SITs in national data systems, the WG made practical considerations on cost, sample size, need for rigorous training and capacity building to employ field studies. Generally, stable isotope-based indicators are relatively expensive due to associated costs like material, logistics and equipment. Second, analysis of stable isotope enrichment in biological specimens is dependent on specialised equipment. Although portable Fourier Transform Infrared Spectrometers are available for body composition and breast milk assessment, the equipment is costly and may not be easily used in the field for large 
surveys. Furthermore, challenges with standardisation of field procedures and practicalities in stable isotope assays have been reported previously [23, 24]. Given these considerations, the WG concluded that SITs are best suited for use in sub-samples of population surveys and for validating other tools to be used in future surveys. The potential for wider scale-up could be more fully explored following validation studies that are incorporated in larger surveys. Moreover, such validation studies offer the opportunity to consider novel methods that are more affordable, field-friendly and possibly include developed handheld measurement equipment.

In summary, the WG identified upcoming national surveys in several countries as opportunities to test the applicability of SITs in population-based nutrition surveillance. It was suggested that SITs should be used in a pilot survey prior to full data collection to validate methods and logistics with higher potential for cost-effective scale-up. For example, deuterium dilution may be used to validate bio-electrical impedance analysis measurements for body composition assessment. The validated bio-electrical impedance analysis can then be applied to a large sub-sample in the actual DHS survey. Another example was to test and validate simpler protocols for assessing exclusive breastfeeding using the DTM technique since the existing protocol requires a 2-week follow-up period for saliva or urine collection. A shorter DTM protocol would reduce both observer and participant burden and may be more cost-effective. One emerging opportunity is the need to validate new tools for body size and volume measurement based on laser technology [25] and handheld 3D scans before they are rolled out for use in large population surveys. The main challenge remains how to ensure standardised and rigorous training in large population surveys involving bigger sample sizes that are typical for studies involving SITs.

\section{Conclusion}

Monitoring of the SDGs and global nutrition commitments based on accurate data is critical. Upscaling the capacity to collect accurate and timely nutrition indicators in the context of large population surveys is needed. Broadening the array of nutritional indicators to include novel aspects that improve evidence-based synthesis and policies and programmes is recommended. With SITs, accurate data on body composition, exclusive breastfeeding and vitamin A status can be collected, eliminating the need for less informative or logistically difficult methods.
Perhaps more important, there is great potential for stable isotopes to be used for the validation of simpler field techniques that can then be used at scale in national household surveys, including DHS. Through this approach, individual countries will have greater capacity to make fully informed policies and programmes that will bring them closer to achieving nutrition-related SDGs.

\section{Acknowledgements}

We are greatly indebted to the following experts for contributing to the discussions of the WG: Merlyn Chapfunga, Nutrition Consultant, World Food Programme, Kingdom of Lesotho; Adelheid Onyango, Regional Adviser for Nutrition, World Health Organization, Regional Office for Africa; Kimberly Ashby-Mitchell, Senior Technical Officer at Caribbean Public Health Agency; Obey Assery Nkya, Prime Minister's Office, United Republic of Tanzania; Jonathan Wells, Professor of Anthropology and Paediatric Nutrition, University College London; Prof Alan Jackson, International Malnutrition Task Force; Prof Anabel Pallaro, Faculty of Pharmacy and Biochemistry, University of Buenos Aires; Angela Padmini de Silva, World Health Organization, South East Asia Regional Office; Christine Slater, Independent Consultant and exIAEA Nutrition Specialist. The authors would like to thank Cornelia Loechl, Daniel Hoffman and Pernille Kaestel for their critical reviews of the manuscript.

\section{Statement of Ethics}

The authors have no ethical conflicts to disclose for this review because there were no humans or animals involved directly.

\section{Disclosure Statement}

The authors alone are responsible for the views expressed in this publication, and they do not necessarily represent the official views of the IAEA. The authors have no other conflicts of interest to declare.

\section{Funding Sources}

No external funding source contributed to the writing of this paper.

Open access provided with a grant from the International Atomic Energy Agency.

\section{Author Contributions}

V.O.O. and T.M. conceptualized the vision and outline of the manuscript. V.O.O. led the drafting of the manuscript, T.M. contributed to writing and both authors contributed to revisions of the manuscript and reviewed the final version. 


\section{Disclaimer}

The statements, opinions and data contained in this publication are solely those of the individual authors and contributors, not of the publishers and the editor(s), and do not necessarily reflect the views of the cooperating organizations, IAEA, UNICEF and WHO. The use of particular designations of countries or territories does not imply any judgement by the cooperating organizations, as to the legal status of such countries or territories, of their authorities and institutions or of the delimitation of their boundaries. The mention of names of specific companies or products (whether or not indicated as registered) does not imply any intention to infringe proprietary rights, nor should it be construed as an endorsement or recommendation on the part of the cooperating organizations.

\section{References}

1 United Nations. United Nations Sustainable Development Goals. [cited Feb 20, 2019]. Available from: https://sustainabledevelopment.un.org/.

2 UNICEF. Brief Note \#3 End hunger, achieve food security and improved nutrition, and promote sustainable agriculture. Nutritional Status 2018. Available from: https://data.unicef.org/wp-content/uploads/2018/05/SDGbriefing-note-3_nutritional-status.pdf.

3 Owino VO, Slater C, Loechl CU. Using stable isotope techniques in nutrition assessments and tracking of global targets post-2015. Proc Nutr Soc. 2017 Nov;76(4):495-503.

4 El Khari K. Expert Mission Report on body composition analysis and interpretation (unpublished). SEY6004: Evaluating an Intervention Program on Obesity and Obesity-Related Risk Factors in Children. Vienna: IAEA; 2018.

5 Williams DP, Going SB, Lohman TG, Harsha DW, Srinivasan SR, Webber LS, et al. Body fatness and risk for elevated blood pressure, total cholesterol, and serum lipoprotein ratios in children and adolescents. Am J Public Health. 1992 Mar;82(3):358-63.

6 Gil L. How Weight in Chile Became a Problem and How Nuclear Techniques Helped. 2017; Available from: https://www.iaea.org/ newscenter/news/how-weight-in-chile-became-a-problem-and-how-nuclear-techniques-helped.

7 Mazariegos M, Slater C, Ramirez-Zea M. Validity of Guatemalan Mother's Self-Reported Breast-Feeding Practices of 3-Month-Old Infants. Food Nutr Bull. 2016 Dec;37(4):494503.

8 Mulol H, Coutsoudis A. Breastmilk Output in a Disadvantaged Community with High HIV Prevalence as Determined by the Deuterium
Oxide Dose-to-Mother Technique. Breastfeed Med. 2016 Mar;11(2):64-9.

9 Medoua GN, Sajo Nana EC, Ndzana AC, Makamto CS, Etame LS, Rikong HA, et al. Breastfeeding practices of Cameroonian mothers determined by dietary recall since birth and the dose-to-the-mother deuteriumoxide turnover technique. Matern Child Nutr. 2012 Jul;8(3):330-9.

10 Oiye S, Mwanda W, Mugambi M, Filteau S, Owino V. Exclusive Breastfeeding Is More Common Among HIV-Infected Than HIVUninfected Kenyan Mothers at 6 Weeks and 6 Months Postpartum. Breastfeed Med. 2017 Jun;12(5):283-9.

11 Herter-Aeberli I, Eliancy K, Rathon Y, Loechl CU, Marhône Pierre J, Zimmermann MB. In Haitian women and preschool children, iron absorption from wheat flour-based meals fortified with sodium iron EDTA is higher than that from meals fortified with ferrous fumarate, and is not affected by Helicobacter pylori infection in children. Br J Nutr. 2017 Aug;118(4):273-9.

12 Loechl CU, Busch T. IAEA's Contribution to Combat Hidden Hunger. 2016.

13 United States Agency for International Development, Demographic Health Surveys.

14 World Health Organization, Noncommunicable diseases and their risk factors: STEPwise approach to surveillance (STEPS).

15 Garrett DA, Sangha JK, Kothari MT, Boyle D. Field-friendly techniques for assessment of biomarkers of nutrition for development. Am J Clin Nutr. 2011 Aug;94(2):685S-90S.

16 Owino VO. Challenges and opportunities to tackle the rising prevalence of diet-related non-communicable diseases in Africa. Proc Nutr Soc. 2019, Epub ahead of print.

17 Owino VO, Murphy-Alford AJ, Kerac M, Bahwere P, Friis H, Berkley JA, et al. Measur- ing growth and medium- and longer-term outcomes in malnourished children. Matern Child Nutr. 2019 Jul;15(3):e12790.

18 Wells JC, Shirley MK. Body composition and the monitoring of non-communicable chronic disease risk. Glob Health Epidemiol Genom. 2016 Oct; $1:$ e18.

19 Wells JC, Fewtrell MS. Measuring body composition. Arch Dis Child. 2006 Jul;91(7):612-7.

20 International Atomic Energy Agency, Stable Isotope Technique to Assess Intake of Human Milk in Breastfed Infants. IAEA Human Health Series; 2010.

21 Jensen SM, Mølgaard C, Ejlerskov KT, Christensen LB, Michaelsen KF, Briend A. Validity of anthropometric measurements to assess body composition, including muscle mass, in 3 -year-old children from the SKOT cohort. Matern Child Nutr. 2015 Jul;11(3):398-408.

22 Duren DL, Sherwood RJ, Czerwinski SA, Lee M, Choh AC, Siervogel RM, et al. Body composition methods: comparisons and interpretation. J Diabetes Sci Technol. 2008 Nov;2(6): 1139-46.

23 Owino VO, Skau J, Omollo S, Konyole S, Kinyuru J, Estambale B, et al.; WinFood Project Team. WinFood data from Kenya and Cambodia: constraints on field procedures. Food Nutr Bull. 2015 Mar;36(1 Suppl):S41-6.

24 Fabiansen C, Yaméogo CW, Devi S, Friis H, Kurpad A, Wells JC. Deuterium dilution technique for body composition assessment: resolving methodological issues in children with moderate acute malnutrition. Isotopes Environ Health Stud. 2017 Aug;53(4):34455.

25 Conkle J, Martorell R. Perspective: Are We Ready to Measure Child Nutritional Status with Lasers? Adv Nutr. 2019 Jan;10 suppl_1: S10-6. 